\title{
Reizgasintoxikation \\ Schnelle Erstbehandlung mit inhalativem Beclometason
}

\author{
H. Buschmann, Übach-Palenberg \\ NOTFALLMEDIZIN 29; 2003: 367-368
}

Seit Anfang 2003 ist das bis dahin einzige bei Reizgasintoxikation zugelassene inhalative Steroid mit Dexamethason aufgrund des FCKW-Verbots nicht mehr verfügbar. Stattdessen wurde vom Bundesinstitut für Arzneimittel- und Medizinprodukte das HFA (Hydrofluoralkan-134a) betriebene BDP-Lösungsaerosol für diese Indikation zugelassen. Zum Einsatz von HFA-Beclometason (BDP) in dieser Notfallindikation liegen kaum publizierte Daten vor. Die vorliegende Kasuistik soll helfen, diese Lücke zu schließen.

$\mathrm{B}$ ei Wohnungs- und Gebäudebränden werden häufig viele für Menschen gefährliche Substanzen freigesetzt, die beim Einatmen auf verschiedene Weise und unterschiedlich schnell eine Reihe von Gesundheitsschäden bis hin zum toxischen Lungenödem hervorrufen können. Brandrauch kann durch seine Temperatur physikalisch das Lungengewebe schädigen, darin enthaltene Giftstoffe wie Kohlenmonoxid (CO) können die Sauerstoffaufnahme in der Lunge blockieren und $\mathrm{zu}$ innerem Ersticken führen. Kohlendioxid $\left(\mathrm{CO}_{2}\right)$ verdrängt den Sauerstoff, Reizgase lassen die empfindlichen Schleimhäute oder gar die gasaustauschenden Membranen der Alveolen anschwellen und behindern so die Atmung. Bei Explosionen kann die Druckwelle Lungenbarotraumen verursachen. Anhand der Symptomatik lässt sich die Art der Schädigung nicht immer zweifelsfrei ermitteln, oft liegen auch Mischformen vor. Deshalb kann eine Erstbehandlung nur relativ unspezifisch und symptomorientiert erfolgen $(1,2)$. Erfahrungsgemäß wirkt sich die Behandlung mit inhalativen Steroiden positiv auf das Befinden von Brandrauch-exponierter Patienten aus. Dieser Substanzgruppe wird unter anderem eine „abdichtende“, schützende Wirkung auf die empfindlichen Schleimhäute zugeschrieben (3), sodass das Auftreten eines Lungenödems verhindert werden kann (4).
Außerdem scheinen inhalative Steroide zu einer schnellen Verbesserung der Ventilation beizutragen. Sie werden in fast jedem Notarztoder Rettungswagen mitgeführt, ihre Anwendung wird bei Rauchgasintoxikationen von den meisten Experten empfohlen.

\section{Studien zu Reizgasintoxi- \\ kationen fast unmöglich}

Die Wirksamkeit einer solchen Maßnahme ist letzten Endes schwer nachzuweisen, da Brände mit Personenschaden nicht sehr häufig vorkommen und niemals vorherzusagen ist, wann und wo sie auftreten werden. Demnach ist es praktisch unmöglich, den Nutzen von inhalativen Kortikoiden unter Studienbedingungen zu prüfen (5). Die Schwierigkeit von Studien an Patienten mit Reizgasintoxikationen ist vermutlich die Ursache dafür, dass ein älteres FCKW-haltiges Dosieraerosol bis Ende 2002 für diese Indikation zugelassen war, während moderne Pulverinhalatoren und HFA-Dosieraerosole nicht eingesetzt werden durften. Seit dem Verbot FCKW-haltiger inhalativer Steroide ab 2003 ist HFA-BDP $\left(\text { Junik }^{\circledR}\right)^{1}$ das einzige für Reizgasintoxikation zugelassene Präparat. Aufgrund der sehr kleinen Partikel kommt es zu einer gleichmäßigen Verteilung des Wirkstoffs bis in die kleinsten Atemwege (6). Darüber hinaus ist die Inhalation mit dem Autohaler ${ }^{\circledR 1}$ auch für Patienten, die

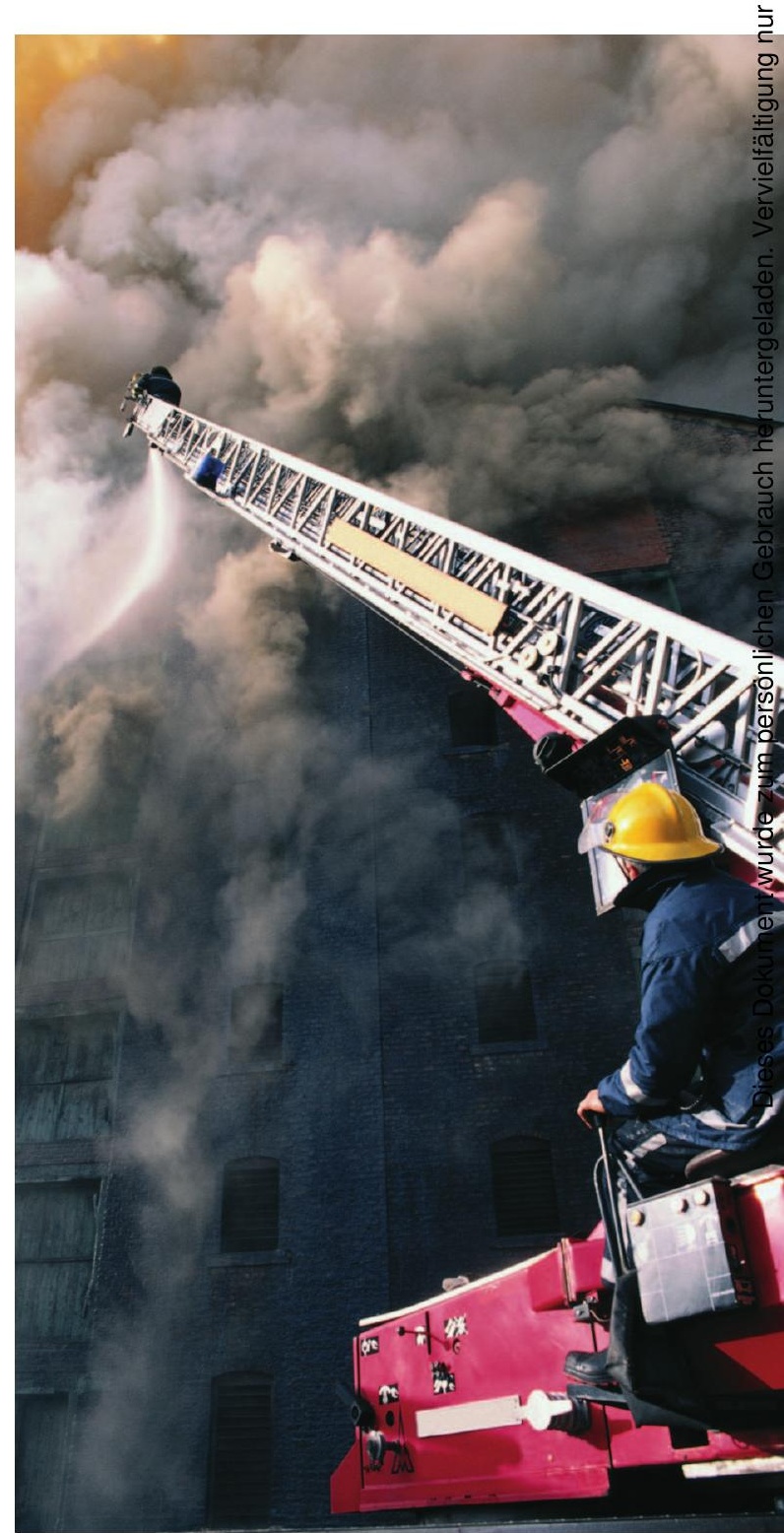


Kasuistik

\section{Tab. 1 Behandlung mit HFA-BDP- Lösungsaerosol nach Rauchgasintoxikation}

\begin{tabular}{rllrl} 
Min. & Hübe & $\begin{array}{l}\mathrm{SpO}_{2} \\
\text { (\%) }\end{array}$ & HF & $\begin{array}{l}\text { PEF } \\
\text { (I/min) }\end{array}$ \\
\hline 1 & 6 & 96 & 132 & 260 \\
2 & 4 & 98 & 104 & 280 \\
5 & 4 & 98 & 98 & 310 \\
10 & 4 & 98 & 102 & 310
\end{tabular}

bisher noch nie inhaliert haben, einfach und schnell zu erlernen (7).

\section{Anamnese}

Eine 52-jährige Hausfrau erlitt am 10.6.2002 eine Rauchgasvergiftung bei einem Garagenbrand mit hoher Schadstoffkonzentration. Freigesetzte Gase waren unter anderem CO (Kohlenmonoxid) und $\mathrm{HCN}$ (Cyanwasserstoff). Die Patientin war unruhig und hatte eine erhöhte Herzfrequenz (134/min). Sie klagte über Brechreiz, Stridor und Globusgefühl.

\section{Befund und Diagnose}

Befund vor der Therapie:

- Sauerstoffsättigung $\left(\mathrm{SpO}_{2}\right): 92 \%$

- Herzfrequenz: 134/min

- Peak Flow (PEF): 260 1/min

Diagnose: Rauchgasintoxikation und Präschock.

\section{Therapie}

Die Patientin inhalierte mehrfach einige Hübe HFA-BDP-Lösungsaerosol. Unter dieser Behandlung und beruhigender Betreuung stabilisierte sich ihr Zustand schnell. Der Peak-Flow stieg innerhalb einiger Minuten deutlich an. Durch die Ventilationsverbesserung und den Beistand fühlte sie sich wieder viel sicherer, Angst und Unruhe gingen zurück. Der genaue Therapieverlauf ist in Tabelle 1 dargestellt.

Nach kurzer Beobachtung konnte die Patientin aus der Behandlung entlassen werden. Drei Tage später war sie vollständig wieder hergestellt.

\section{Diskussion}

Anhand der Symptome einer Rauchgasvergiftung lässt sich das Ausmaß der Lungenschädigung nicht immer leicht erkennen. So ist es in der akuten Notfallsituation nicht einfach festzustellen, ob eine Schleimhautreizung der oberen Luftwege oder Schädigungen der kleinen Atemwege vorliegen, oder ob eine Mischform besteht. Für die schnelle Erstbehandlung sind daher Strategien gefragt, die relativ unspezifisch zunächst eine Verbesserung der Ventilation und die Beruhigung des Patienten bewirken, um nach einer kurzen Beobachtungsphase zu entscheiden, ob weitere Maßnahmen, wie zum Beispiel der Transport in eine Klinik, notwendig sind $(5,8)$.

Der Zustand der in der Kasuistik vorgestellten Patientin stabilisierte sich unter der Behandlung mit dem HFA-BDP-Lösungsaerosol. Es lässt sich aufgrund der vorgegebenen Randbedingungen einer Notfallbehandlung nicht eindeutig nachweisen, ob sich die Atmung der Patientin vorwiegend durch das inhalierte Steroid stabilisiert hat. Dies ist jedoch zweitrangig, solange insgesamt ein objektiver Nutzen erkennbar beziehungsweise zu erwarten ist (5). Psychische Faktoren oder die Erholung an der "frischen Luft" nach der Rettung aus dem Gefahrenbereich spielten vermutlich auch eine Rolle. Jedoch haben Glukokortikoide, neben ihrer rezeptorvermittelten antientzündlichen Wirkung, einen direkten membranstabilisierenden Effekt. Dadurch wird die entzündungsbedingte Sekretion sowohl im Gewebe als auch in den Alveolen reduziert. In vielen Fällen kann so durch inhalative Steroide ein Lungenödem verhindert werden (3).

In der Regel nehmen die mit HFA-BDP Behandelten eine Linderung ihrer Atembeschwerden durch die inhalative Therapie wahr. Nach meiner Beobachtung wirkt sich die sofortige Therapie mit dem HFABDP-Lösungsaerosol messbar positiv auf die Ventilation von rauchgasexponierten Patienten aus. Publizierte retrospektive Analysen bestätigen einen entsprechenden Effekt (9).

\section{Summary}

Chlorofluorocarbon(CFC)-containing formulations of inhaled corticosteroids were taken from the market in the beginning of 2003. The only glucocorticoid containing metered dose inhaler admitted for use after poisoning by smoke inhalation and accidental exposure to gases contained CFC and is no longer available. In order to fill the gap, hydrofluoroalkan-beclomethasone dipropionate (HFA-BDP) aerosol was approved. HFA-BDP has been used effectively for many years for the treatment of asthma. Since clinical studies are not available for the emergency application a case description offers rationale and shows the efficacy and administration procedure of HFA-BDP.

\section{Literatur}

1. Möllmann H et al. Differential therapeutic aspects of the treatment of toxic gas poisoning with glucocorticoids. Intensivmed. 1989; 26: 2-15

2. Ortner; Heuseler. Beurteilung von Kunststoffbränden. Bayerisches Landesamt für Umweltschutz

3. Kaiser H, Kley H. Cortisontherapie. Corticoide in Klinik und Praxis. Stuttgart-New York: Georg Thieme Verlag, 1997

4. Vogel F. Inhalierbare Corticoide als Prophylaxe zur Therapie inhalierbarer Noxen. Notfallmedizin 1985; 11: 1447-1456

5. Lechleuthner A, Steffens W. Cortison bei Reizgasinhalation. Wissenschaftliche Arbeiten des Instituts für Notfallmedizin der Berufsfeuerwehr Köln, 1999

6. Leach CL. Improves delivery of inhaled steroids to the large and small airways. Respir Med 1998; 92 (Suppl. A)

7. Cegla UH. Optimierung der Inhalationstherapie - Autohaler ${ }^{\circledR} \&$ Co. Topmedizin 1996; 10 (03): 2-9

8. Peibst H, Krohn S. Gefahren an der Einsatzstelle. Berufsfeuerwehr Halle Süd

9. Xie L et al. Analysis of the treatment of 82 patients with inhalation injury. Zhonghua Zheng Xing Shao Shang Wai Ke Za Zhi 1999; 15 (6): 414-416

\section{Anschrift des Verfassers}

Dr. med. Heiner Buschmann

Facharzt für Allgemeinmedizin, Feuerwehrarzt

Merksteiner Straße 2

52531 Übach-Palenberg

${ }^{1}$ Fujisawa Deutschland GmbH 